\title{
Retrieval of Motion Capture Data Aids Efficient Digital Learning
}

\author{
Sheng-Chih Chen ${ }^{\mathrm{a}}$, Wei-Kuang Chen ${ }^{\mathrm{b}}$, Tsai-Sheng Kao ${ }^{\mathrm{c}}$, Jui-I Hsu ${ }^{\mathrm{d}}$ \\ ${ }^{a}$ Master`s Program of Digital Content and Technologies, College of Communication, National ChengChi, \\ University, Taipei City, Taiwan, R.O.C. \\ ${ }^{b}$ Department of Architecture, National Taiwan University of Science and Technology Taipei City, Taiwan, \\ R.O.C. \\ ${ }^{c}$ Department of Electronic Engineering, Hwa-Hsia Institute of Technology, Chung Ho, Taipei County, Taiwan, \\ R.O.C. \\ ${ }^{d}$ Department of Digital Multimedia Arts, Shih Hsin University, Taipei City, Taiwan, R.O.C.
}

\begin{abstract}
This study aims to integrate digital technology with the animation production process. By experiencing and learning how body joints move - with digital technology as the learning aid - students can create results similar to motion-captured body movements. This then can be applied to animation design with the hope that it can help the students in their future employment. This paper focuses on digital learning and technology to bring forth body movement production principles and an integrative framework. The study results can offer training to front-end talents of the digital content industry. In addition, the main contribution lies in the research and development of training methods and linking them with digital learning techniques. This can provide directions for the development of upcoming relevant cultural industrial courses. This paper will use human-like character animation - that is comparatively harder to represent in 3D computer animation - as the example. It will also discuss the variations in results achieved by different production processes. At the same time, feasible training directions are provided as references for learning digital technologies.
\end{abstract}

Index Terms: Cross-disciplinary integration; digital learning; human-like characters; motion capture

(C) 2012 Published by MECS Publisher. Selection and/or peer review under responsibility of the International Conference on E-Business System and Education Technology

\section{Introduction}

Digital contents today are all-embracing, including audio visual entertainment, information communication, education, learning, and business services; all can be carried out via the Internet or other digital platforms. At the same time, the application of computer animation in digital content has shown continuous improvement in both quality and quantity. Over the last ten years, the role of 3D computer graphics in animation has gained importance. This is because the progress in calculation ability, 3D modeling tools, presentation devices, scanning technology, and virtual reality - combined with other aspects of technologies — has resulted in accurate

* Corresponding author.

E-mail address: ${ }^{\mathrm{a}} \mathrm{scchen} @$ nccu.edu.tw, ${ }^{\mathrm{b}}$ p6109@ms24.hinet.net, ${ }^{\mathrm{c}}$ tskao@cc.hwh.edu.tw, ${ }^{\mathrm{d}}$ ray3dsf@ gmail.com 
representation of buildings, antiquities, human emotions, and even the ecological system. Applying computer technologies, such as image processing, computer graphics, solid modeling, rendering, and animation, would deliver more diverse visual experiences of human-like characters. These technologies even can assist in simulation of simultaneous human characters and scenes.

Accompanied by the rapid progress of 3D computer imaging technology, human-like character animation with a motion capture system and the ability to move virtual characters in three-dimensional virtual spaces has evolved into the trend of motion applications. When applied to the movie industry, virtual technologies have a significant effect on movies. A motion capture system, by which the motion of real actors is filmed and applied to virtual characters, enables the motion of virtual characters to be as elastic as that of real humans [8]. In computer animation, motion capture is today's most comprehensible and discussed technology [3][4][5][6].

The policy concerning Cultural \& Creative Industry Courses (CCIC) in universities established responds to the industrial development. Therefore, by observing from the sequence of subjects started combined with each industry, there is no practice of courses that links dancing or performing act with the simulation of newly emerged technologies. Reasons for this include inadequate equipment, uncertainty to how cross-disciplinary integration can be made, and the unmet expectations of the technologies applied in digital learning technology. Thus, this study places its emphasis on how to research and develop relevant courses that enable students to learn the skills matched with the industrial demands during their schooling period. In addition, it is already clear that the core topics of this study are related to the decoding and application of human body movements. On the premise of course research and development, the following should be considered. First, designers need to become familiar with the relationship between human body movements and bone and joint operation before they are able to penetratingly produce animation movements that contain no visual errors or illogicality. Second, the prior coordination and communication between actors /performers and designers constitute the primary key to the creativity and effect representation.

Information age offers brand-new creative themes, methods and thoughts which assist designers to quickly satisfy the spatial demands and easier representation of spatial concepts. The spatial cognition also gradually blurs out following the increasingly realistic simulating images. Nevertheless, the images and interaction form the closest relationship attached to virtual space and human beings. As a result, visualization and interactiveness are used as the based research factors. Although the rapid changes in the computer animation production software and hardware greatly lowers the learning threshold of relevant knowledge and skills, the difficulty in production still exists for some computer animation contents of specific categories. This paper will use humanlike character animation -- that is comparatively harder to represent in 3-D computer animation - as the example. It will also discuss the variation in the results reached by different production processes. At the same time, feasible training directions are provided as the references for the learning of digital technologies.

\section{Modeling and Motion Capture Techniques}

\section{A. Creating Human-like Character Animation with Motion Capture System}

The question of what is human-like character animation involves the definition and scope of character animation, the features of 3D human-like characters, and the distinction between 3D human-like characters and cartoon animation characters. In human body language, body motion principles, the connection between behaviors and emotions, and the artistic interpretation of body performance all are essential for the exploration and expression [2][3][5][6]. To produce motion of virtual characters, three main categories of tools are included: keyframe (hand-made) production, motion capture, and artificial intelligence (AI) motion simulation tools.

From the 2000s up to present, technologies have realized the symbolization of national power in information. Wireless sensor system, intelligent hand-held devices and embedded systems, and immersed digital technology virtual reality are a few examples that have opened up a new prospect of computer technologies. Among these technologies, motion capture systems started to change the ways by which businesses carry out their marketing, design and product manufacturing activities. Advertisers dream to be able to use motion capture to stop the consumers' tracks. The changes in the displacement, rotation and zooming of the detecting cameras transmit the 
data to the computer in real-time while the computer updates the changes on the screen rapidly and synchronously. The unique features of motion capture systems, such as rapid alterations of different films, convenient modifications to scenes, no restrictions to the current construction techniques and materials and the strong interaction with the filming set. Different tracking methods could even been derived to verify the absolute coordinates and relative coordinates of the cameras, scenes, and performers/actors in the space.

This study draws on an optical motion capture system of Visualeyez which differs from other motion capture systems in the following ways: since each led target has an independent number assigned to it, identification errors and data misplacement will not happen during tracking, unlike other optical motion capture systems. This characteristic eliminates the need to re-identify the number of the marker manually during subsequent data processing, which also lowers the difficulty in data identifying after the led targets are hidden and saves working time.

The system used in this study has fast tracker calibration, which can normally be complete within ten minutes (exclusive of actor calibration). It saves time and effort and can be operated by one person alone. When only one set of tracking device is used, calibration may be skipped. If more than four sets of tracking devices are used, two actors' motion can be captured simultaneously. The tracker is not fixed and it can adjust its position according to the motion of the actor's requirement and can move to another suitable location for motion capture. It supports real-time streaming function. When actors are performing motions, through plug-in function, virtual actors can display motion results synchronously in other motion data processing software. The performing condition of actors can be observed right away, which does not need to be postponed to the post-production. This facilitates immediate decision to be made regarding whether any change or redoing is required at the film set.

\section{B. Making Human-like Character Animation Using Keyframe}

When learning character animation, it is important to understand the fundamental attributes and characteristics of the moving objects in animation software, to control the relationship between time and distance and the coordination of body language (ability to do body sketching), and to comprehend the overall concept of the basics of moving body parts. This is essential for developing the ability to demonstrate the beauty of character motions, to construct a character skeleton, and to set the inverse controller in animation software and perform the tests required before character animation films are completed.

The 12 basic principles of animation are brought together by Disney animators based on their experiences accumulated over several dozen years [7][9]. These principles that can and should be applied to 3D computer animation are: 1.Squash and Stretch; 2.Anticipation; 3.Staging; 4.Straight-ahead vs. Pose-to-pose; 5. Followthrough and Overlapping Action; 6. Slow-in and Slow-out; 7.Arcs; 8. Secondary Action; 9.Timing; 10.Exaggeration; 11.Solid drawing; and 12. Appeal. These principles and the kills required to articulate them are important to those who want to become successful animators.

Murakami, Nakamura, \& Kuno introduced a system to produce digital contents of traditional dances for digital archives, integrating motion data acquired from a motion capture system and multiple video images. This system makes it possible to analyze the ways each body part moves in video images, even when the actors are wearing loose garments that prevent the viewer from seeing under them[11]. On the other hand, Tabata, Nakamura, and Kuno described the development of active devices which were worn on the body in order to teach dance motion. The goal was to construct a basic dance training system that would help the beginning dancer easily learn the basics of traditional dances. Images were used to provide the learner with dance information. The system incorporated a mobile robot with a display mounted on its head. The learner could watch a fixed screen and the display mounted on the head of the mobile robot while the robot completed the dance steps. In addition, the system allowed an analysis to be made for the differences between data collected about dance instructors and the learners, which was then transmitted to the devices. The learner was given this information by vibration. Using these active devices, learners could experience dance instruction directly and master the dance steps efficiently [10]. Furthermore, Jabłoński, Klempous and Majchrzak explored the possibilities of identifying a person via leg rotation signal analysis when amplitude spectrum analysis and a Dynamic Time Warping method (DTW) were 
applied [1]. Their research described the possibilities involved in the application of a variety of motion analysis methods and offered suggestions for directing the advancement of biometric identification.

\section{Methodology and Steps}

\section{Experiment Description and Equipment Requirements}

The first experiment target was a master's student at the Graduate School of Digital Industries with five years of animation experience. The second experiment target was a master's student at a graduate school of dance with 15 years of dance experience. To study the integrated methodology and steps, dance was used as the example in combination with the operation methods and processes of animation production. The experiments carried out validated the process time and result quality. A comparison of the training strategies confirmed the feasibility and effectiveness of cross-disciplinary training course design with improved training methods to be utilized in industrial cooperation. Several important issues would be discussed, such as the limitation and flexibility between bones and muscle kinematics (within the scope of in-depth understanding and free operating mechanism), and the free control techniques of the angles of skeleton joint objects in computer animation. Experiments were categorized in two groups: Motion Capture group (Group A) and Keyframe group (Group B) (Table 1).

TABLE I. EXPERIMENT GROUPS AND OPERATION CONTENT

\begin{tabular}{|c|l|l|}
\hline & \multicolumn{1}{|c|}{$\begin{array}{c}\text { Motion Capture } \\
\text { Group (Group A) }\end{array}$} & \multicolumn{1}{c|}{$\begin{array}{c}\text { Keyframe Group } \\
\text { (Group B) }\end{array}$} \\
\hline Method & $\begin{array}{l}\text { Acquire motion data } \\
\text { from motion capture } \\
\text { system }\end{array}$ & $\begin{array}{l}\text { Acquire data through } \\
\text { character motion process } \\
\text { and apply to the comparison } \\
\text { with keyframe in animation } \\
\text { software (virtual character } \\
\text { motion) }\end{array}$ \\
\hline Practice & $\begin{array}{l}\text { Use the software and } \\
\text { hardware specific to } \\
\text { an optical motion } \\
\text { capture system }\end{array}$ & $\begin{array}{l}\text { Install three virtual cameras } \\
\text { in one computer }\end{array}$ \\
\hline
\end{tabular}

In this study, the Visualeyez optical tracking device developed by the Canadian firm Phoenix Technology Inc. (PTI) was employed. Because the software used in a motion capture system cannot produce 3D computer animation, it is necessary to use matching software to accomplish the 3D character geometry, material, and skeleton system. To accomplish motion data modifications and other editing work, OEM software alone is insufficient. A software product specifically designed for motion data processing, such as FilmBox or MotionBuilder, is used to complete the post-production work on files and converting motion data format.

\section{Experiment Results and Operation Process}

Experiment results obtained from the operation process are shown in the following pictures: Setting up equipment and location; motion capture software, and prior calibration and motion capture (shown in pictures left to right, Figure 1). 


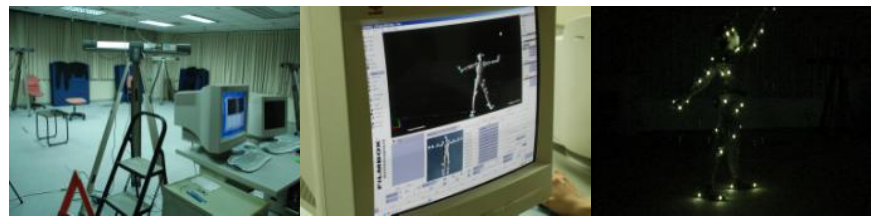

Figure 1. Experiment A Process

The dancer's demonstration and training instruction and the designer's experience in learning; virtual character establishment and comparison with motion film (Figure 2).

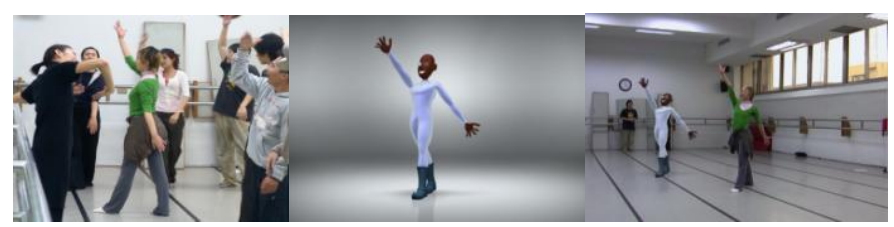

Figure 2. Experiment B Process

The training design and production process of the two groups are presented below (Figure 3 and 4):

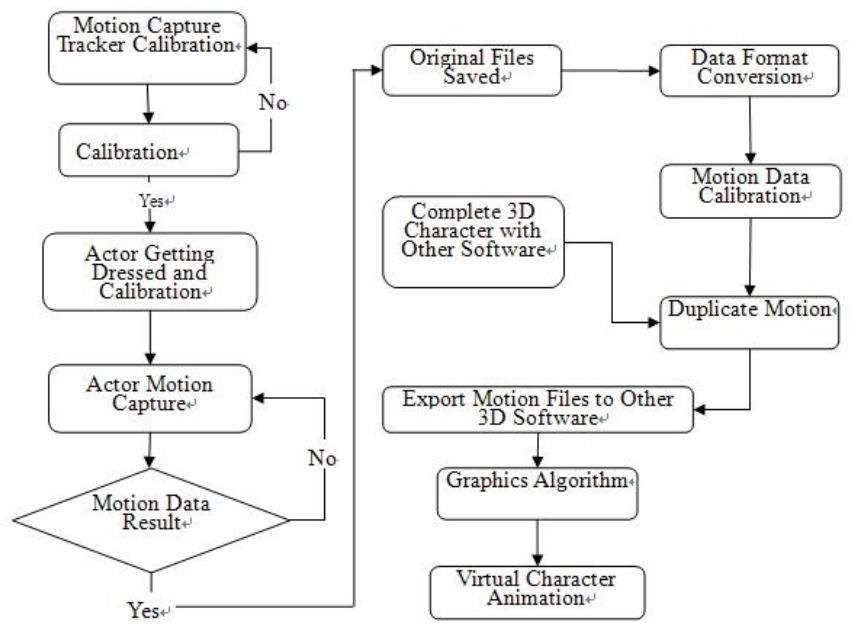

Figure 3. Experiment A Group (motion capture group, Mocap Group) 


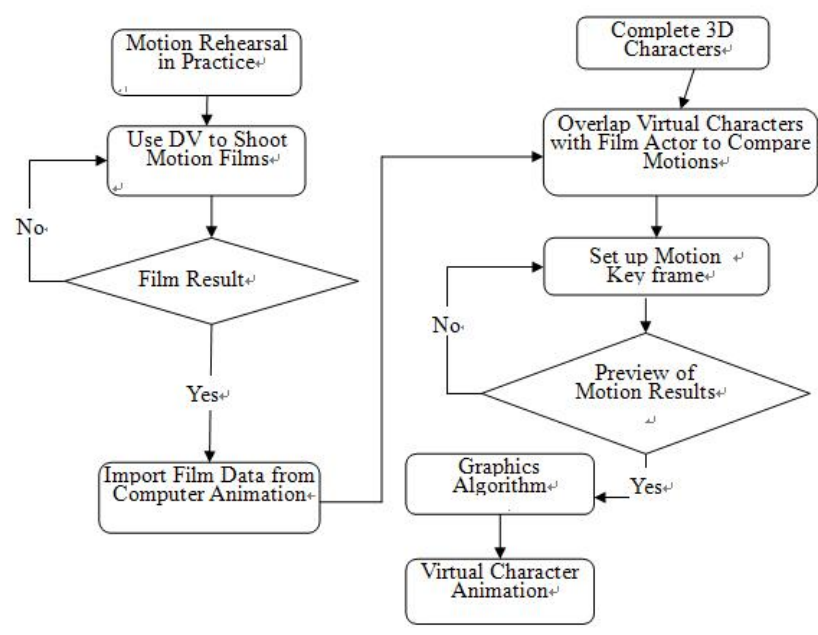

Figure 4. Experiment B Group (keyframe group)

\section{Training Course Analysis and Discussion}

\section{E. Motion Capture Training Course Research}

1) Advantages of Motion Capture System in Computer Animation and Game Production Training

a) THE APPEAL OF TRAINING COURSE TO STUDENTS

Digital media today is very well-developed; students can easily collect various types of information regarding computer animation and game production. Since motion capture has been used on the entertainment content related technologies engaged in movie industry, game production and movie special effects.. Students who learn relevant specialized knowledge are eager to experience the power of this tool firsthand. Thus, the courses will appeal to the students very easily and trigger their learning motive and willingness. Take the "Motion Capture and Editing Technologies" offered in the Department of Digital Multimedia Arts of Shih Hsin University, as the example, the number of students who studied it as an elective subject during the first stage since its commencement in 2005 exceeded the limit. This shows the students' high degree of interest in that course.

\section{b) The Effect of Finished Products on Sense of Achievement in Learning}

Whether it is difficult to create 3-D virtual character motion by using keyframe is difficult or not relies on the students' level of understanding about motion analysis and animation concepts. It is also restricted by the design of animation software in animation production module. If it is difficult to operate or learn the software, the students who have deep interests in elimination learning will suffer the setbacks which will lower their learning willingness to learn.

With appropriate training plans, the learning process of motion capture system may not be as fast, but students can have greater satisfaction in the products finished via this tool. In view of ordinary students who produce character animation by using keyframe, these students usually do not possess sufficient experience. The results of achieving real but complex motion of real people were usually inferior to the motion capture data performed by real actors. If coupled with motion capture data, and students use keyframe to adjust some of the motions enable them to produce smoother and more lifelike character animation. This does not only increase student production quality, but can also enhance the students' sense of achievement. They will feel more confidence in their production ability that benefits their learning willingness.

c) The Competitive Advantages of Product Presentation 
Visual presentation occupies an important part in final animation and game production work. The audience with no expertise in this background offers the standard for direct judgment. The good or bad of virtual characters in motion presentation affects the appraisal and reception of the audience about the work on the spot. Since most of the products in the industry demand rigorous visual presentation and excellent standard, this circumstance can place great pressure on the creators/producers who are still at their learning stage. However, this also motivates them to improve on their production work standard. If the students can effectively draw supports from a motion capture system to produce high quality motion data and optimize the application results of the data, this can definitely add values to the visual presentation of the animation and game created on one's own.

\section{2) Related Issues on Motion Capture Training Course}

\section{a) Budgets for Motion Capture Studio Configuration}

The budgets for motion capture studio configuration consist of the purchase of software and hardware. The cost for purchasing hardware ranges from a few million to ten million New Taiwan dollars. This constitutes a huge burden to a school's budgeting. In addition, many motion capture systems can only operate within an environment similar to that of a photographic studio. The space planning may possibly add to the difficulty of configuration.

For training software, at times appropriate supporting software programs are required in order to proceed with motion data postproduction. Purchasing the software will incur higher costs, but not all the motion capture systems have such a request. Furthermore, the computer equipment that deals with motion capture information must meet certain level of hardware standard, such as high-performance video adaptor and faster system processor. If the equipment is lower in standard, additional expenditure on hardware upgrade needs to be paid.

\section{b) Training Plans and Instructors}

For classes containing a large number of students, they can only be divided into groups. In a group with five or six students, work can be appropriately distributed according to the jobs involved: Actor, who is responsible for performing motions appointed on the motion schedule or script. Motion Director, must understand the script structure and motion requirements very well. When the actor has any performing problem, he or she can make recommendations and help the actor to complete the shooting of motions. Project Leader is responsible for the control of the entire project progress. If there is problem in performance, he or she has to coordinate with actor and motion director, which will result in a decision taken regarding whether to accept or retake the shooting. The operator, responsible for operating the computer system, needs to take care of the starting and stopping of a motion capture system, record and save motion capture data in computer software. The operator needs to work with the Project Leader to examine the data quality.

\section{3) Training Course Schedule and Assessment}

\section{a) Course schedule:}

Take the example of the elective course, "Motion Capture and Editing Technologies "offered at the Department of Digital Multimedia Arts of Shih Hsin University for students in their third academic year or above, the course is divided into two semesters for the first stage (basic) and the second stage (advanced). The course covers motion capture principles and system introduction, motion capture studio hardware configuration, motion capture studio practice, concepts of motion data processing and software description, motion data calibration, character motion adjustments, character motion exporting and file conversion, motion design analysis, and group practices in motion capture projects. It would take at least 3-13 weeks of training to allow a student to complete this dance motion capture experiment project. It also takes no less than 20 hours of classroom and practice training for a person to be qualified to produce the project results. This figure does not include the training of 3-D character model construction. Time spend on modeling, texture mapping and skeleton system production are not part of the course contents mentioned above so they will not be calculated.

b) Training Assessment Referenc 
By conducting an assessment of the training course, observation on the suitability of course arrangement and implementation is possible. Students feedback can be obtained to adjust the contents for teaching. Shih Hsin University uses a questionnaire to conduct assessment. The questionnaire has 32 questions and are classified according to the following categories: 1) teaching content related; 2) the instructor and the teaching performance; 3) course assessment related 4) student self evaluation; and 5) others. Among the five categories, teaching content related questions have the most referential values for course design and implementation results.

\section{F. Character Animation Training Strategies}

In character animation production, there are distinct differences between the two production approaches of traditional hand-drawn animation image and computer images. Since computer graphics is based on data computation and parameter control, many commands and functions in the software have been designed to stay very close to the perfect laws of physics in the real world. It is also positioned as a tool for creativity, which enables the user to produce the arcs generated by animation production using self defined abstract conditions. For novices who have never experienced 3-D computer animation software before, they would feel the inability to manipulate such flexible mechanism. A description for teaching the concepts and techniques related to 3-D computer animation software in character animation production will be provided below.

1) Understanding the Fundamental Attributes and Characteristics of Moving Objects in Animation Software.

In computer animation software, the first thing to learn in computer animation is to make a virtual object "move". Beginners need to know the characteristics of the objects in motion, described as follows: 1 . Only linear motion of a virtual object will be present between the two moving keyframes in animation software. 2. For an object to show curved motion, key nodes must be added at locations outside the liner motion of the object within the two moving keyframes. In this way, the relationship curve can be generated for the animated tracks of first and second particles (front and back). In fact, the patterns of motion for the majority of objects in the natural world consist of to simple linear motion as well as path motion in circular arc. There are also uniform speed motions or variable speed motions. Comprehensively speak; making Arcs animated tracks is the main fundamental concept in animation among the 12 animation principles.

\section{2) Grasping the Relationship between Time and Distance}

Producing character animation is much more complicated than the simple linear or curved motion of an object. It is harder to observe or imitate the detailed changes in motion for the former. Making use of the "Straightahead and Pose-to-pose" among the 12 animation principles is a comparatively better method. Producing too many keyframes in the very beginning is not a smart animation production method. The appropriate way is to determine the average keyframe within one set of motion or alternatively find the largest turning point. Utilizing a comparatively longer time range can make important Key Poses.

\section{3) Kinematical Coordination of Human Body Language (Body Sketching Ability)}

During the process of boy movement, how can one find the key body pose? Through observation and hand drawn imitation of the human body movements, the students can be trained on the motion analysis ability and acquire an in-depth understanding of the balance between static and dynamic states of various human body postures. It is similar to the character motions in comic strip storyboard form that set off the changes in time and space.

\section{4) Understanding the Basics of Moving Body Parts to Demonstrate Aesthetic Perception}

With reference to videos of dancer performance, students who perform motion results according to one's wishes can personally feel the rhythm of the body movements in dance. For example, if a learner of animation production can study the dance steps in the script, an animator can actually recognize the relationship between the each rotation angle of the joints and muscle movements of a virtual character. The learner can also experience the expected form of beauty from the correlation among the rhythm of character movements, time changes and spatial state. 


\section{5) Character Skeleton Construction in Animation Software and Set-up of Inverse Controller}

The construction method of 3D computer animation in attempting to simulate actual character skeleton is very cautious. The positions of skeleton joints are defined in accordance with the skeleton anatomical diagram in medicine. Producing the skeleton structure usually uses the point below the intersection of pelvis and the spine (where the center of the pelvis is above the midway of the thighs) of the character as the starting point. This is extended to the tips of the limbs and the top of the head. When operating the skeleton posture in animation software, there are two common control structures:

\section{a) Forward Kinematics (FK)}

It implies the normal movement method when human bodies are in motion. The total motion volume increases progressively when moving down to the lower parts. For example, to raise a hand requires the rotating of the upper arm, then the front arm, and last the wrist. When rotating the upper arm, the body parts connected move together.

\section{b) Inverse Kinematics (IK)}

Inverse Kinematics is more like controlling puppets. Puppets usually have the strings tied to the ends of their arms and legs and the body. Pulling the strings can control their motion. Computer animation software can use the setting of inverse controller to restrain the joints from shoulder blade to wrists, thigh bone to the ankles, and other connectable joints. In this way, motion can be generated by pulling the ends of the body parts.

\section{6) Testing Before Completion of Character Animation}

After motion adjustment is done using keyframe, software will be used to compute a raw film footage. This is because motion is played with varied speed in the software which is not necessarily real-time. Sometimes, the effects of computer efficacy or file size may also cause errors. Therefore, when the motion production comes to an end, motion results will be quickly computed as a video file of low resolution in order to observe if the motion is accurate under normal playing speed.

\section{Conclusions}

The experiments with the two groups verified that, without a doubt, the data gathered by the motion capture system showed greater precision and smoothness. However, improved digital learning methods and training strategies do not only allow students to experience actual body movements but also adjust the motion of virtual characters by keyframe. Greater smoothness could be achieved relative to the previous traditional animation production method. Using inverse control on the character skeleton in motion would allow for the determination of joint complex automation at a certain angle. This effect could be generated from the point of view of character animation learning and training strategies. If a learner could produce these keyframes timely and abstractly, the character in animation could react freely in a continuously changing environment. Not every school has the equipment for students to learn emotion capture and production techniques and schools have difficulty expanding their budgets. This inadequacy, however, can be remedied through course design. Students could experience the production practice in the industry during the operation process and actually learn this skill.

\section{Limitation, Significance and Future Works}

The extent of the applications of human-like character animation in the digital content industry cannot be ignored. This study has two subsequent directions. First is to set up a digital learning database of the relevant skills required for using keyframe technique. Second, an automatic generation system will be established that transcribe the data acquired from motion capture into programming language. This can be applied not only to robot research but also to the creation of a fast mechanism for animation generation.

This study investigated the structural and operational changes in digital learning methods in order to provide solutions to overcome schools' difficulty in acquiring motion capture equipment and expanding budgets. In this 
way, students could reach the threshold of learning motion capture and production techniques. This study has proposed that digital learning courses and training strategies should be designed with the hope of overcoming any limitations. This could strengthen the skills students acquire, which would provide them with value-added contribution. They would be well-equipped to enter the workforce and meet future industrial demands. In the analysis of character animation, this study has not touched upon the detailed motion changes involving emotions, fingers, and toes. Future research should explore this topic.

\section{References}

[1] B. Jabłoński, R. Klempous, and D. Majchrzak, "Feasibility Analysis of Human Motion Identification using Motion Capture," proceedings of the 25th IASTED international conference on Modeling, identification, and control, 2006, pp. 495-500.

[2] J. Davis and A. Tyagi, "A Reliable-Inference Framework for Pose-Based Recognition of Human Actions," in IEEE International Conference on Advanced Video and Signal Based Surveillance, Miami, Florida, July 21-22, 2003.

[3] J. Davis and V. Kannappan, "Expressive Features for Movement Exaggeration," SIGGRAPH Conference Abstracts and Applications (Technical Sketches), San Antonio, Texas, July 24, 2002, pp. 182.

[4] J. Davis, and H. Gao, "An Expressive Three-Mode Principal Components Model of Human Action Style," Image and Vision Computing, Vol. 21, No. 11, 2003, pp. 1001-1016.

[5] J. Davis, and H. Gao, "Gender Recognition from Walking Movements using Adaptive Three-Mode PCA," IEEE Workshop on Articulated and Nonrigid Motion, Washington DC, June 27, 2004.

[6] J. Davis, and H. Gao, "Recognizing Human Action Efforts: An Adaptive Three-Mode PCA Framework," International Conference on Computer Vision, Nice, France, Oct 13-16, 2003, pp. 1463-1469.

[7] J. Lasseter, "Principles of Traditional Animation Applied to 3D Computer Animation," ACM SIGGRAPH Computer Graphics, vol. 21, no. 4, 1987, pp. 35-44.

[8] K. Pullen and C. Bregler, "Motion capture assisted animation: texturing and synthesis," ACM Transactions on Graphics, vol. 21, iss. 3, 2002, pp. 501-508.

[9] S. Owen, Computer Animation Website. http://www.siggraph.org/education/materials/HyperGraph/animation/anim0.htm, 2002.

[10] S. Tabata, A. Nakamura, and Y. Kuno, "Development of an Easy Dance Teaching System using Active Devices," proceedings of the IASTED International Conference on Advances in Computer Science and Technology (ACST 2004), 2004, pp. 38-43.

[11]T. Murakami, A. Nakamura, and Y. Kuno, "Generation of Digital Contents for Traditional Dances by Integrating Appearance and Motion Data," proceedings of the Second IASTED International Conference on Visualization, Imaging, and Image Processing, 2002, pp. 672-676. 